\title{
Modelocked Tm-doped Fiber Laser Using Multimode Interference Saturable Absorber in Reflection Mode
}

This paper was downloaded from TechRxiv (https://www.techrxiv.org).

LICENSE

CC BY 4.0

SUBMISSION DATE / POSTED DATE

10-02-2022 / 11-02-2022

CITATION

Rochette, Martin; Anjum, Arslan (2022): Modelocked Tm-doped Fiber Laser Using Multimode Interference Saturable Absorber in Reflection Mode. TechRxiv. Preprint. https://doi.org/10.36227/techrxiv.19153865.v1

$\mathrm{DOI}$

10.36227/techrxiv.19153865.v1 


\title{
Modelocked Tm-doped Fiber Laser Using Multimode Interference Saturable Absorber in Reflection Mode
}

\author{
Arslan Anjum and Martin Rochette, Senior Member, IEEE
}

\begin{abstract}
We present a reflection mode saturable absorber based on nonlinear multimode interference in a graded index multimode fiber. The saturable absorber provides a modulation depth of $12 \%$ at a low saturation intensity of $18.5 \mathrm{MW} / \mathrm{cm}^{2}$. The all-fiber structure and reflection mode design of this saturable absorber makes it an ideal device for the fabrication of fiber lasers with linear resonant cavity. When inserted in a linear laser cavity comprising a thulium doped fiber as the gain medium, the saturable absorber triggers modelocked noise like pulses with a center wavelength of $1905 \mathrm{~nm}$ and spectral width of $11.2 \mathrm{~nm}$.
\end{abstract}

Index Terms-Modelocked Fiber Laser, Nonlinear Multimode Interference, Saturable Absorber

\section{INTRODUCTION}

$\mathrm{P}$ assive saturable absorbers (SAs) are essential building blocks that trigger a pulsed regime in lasers. SAs based on semiconductor mirrors [1], graphene [2], nonlinear polarization rotation [3] and multimode interference (MMI) [4] have been employed to create modelocked fiber lasers at various wavelengths. MMI based SAs are particularly desirable for fiber lasers due to their all-fiber design and compatibility for modelocking over a broad wavelength range. In addition to the common generation of a single soliton pulse at cavity roundtrip rate, passive SAs have also enabled generation of other output pulse formats such as noise like pulses (NLP) [5], soliton rain [6] and rogue waves [7]. NLP are modelocked pulses characterized by a duration of the order of the picosecond to nanosecond, with random femtosecond features. Furthermore, they have a smooth and broad averaged optical spectrum and low coherence time [8], [9]. These properties make NLP especially attractive for applications like low coherence spectral interferometry [10], [11], supercontinuum generation [12] and micromachining [13].

A typical MMI-SA consists of a multimode fiber (MMF) that is sandwiched in between input and output single mode fibers (SMF). In the MMF, excited modes experience an intensity dependent relative phase shift that results in a SA transmission response. The characteristics of a MMI-SA such as saturation

This paragraph of the first footnote will contain the date on which you submitted your paper for review. It will also contain support information, including sponsor and financial support acknowledgment. (Corresponding author: Arslan Anjum.) threshold, modulation amplitude, and central wavelength can be varied from adjustment of the MMF length as well as fiber to fiber alignment to modify the coupled power to/from various MMF modes [4]. A precise adjustment of the MMF length is hard to implement due to the short imaging length of the MMF e.g., $\quad 1.15 \mathrm{~mm}$ for typical graded index MMF [14]. Alternatively, to adjust the coupled power to/from MMF modes, mode adoption [15], bending [16] and offset coupling [17] have been demonstrated.

To date, all the MMI-SAs have been reported in a transmission mode and are thus better designed for use in ring resonant cavities. In contrast, it would be advantageous to implement an all-fiber MMI-SA that operates in a reflection mode to accommodate situations when a linear resonant cavity is most desirable. For example, to avoid the use of optical isolators or circulators needed to ensure the optical stability of circular laser cavities. An all fiber MMI-SA that operates in reflection mode is therefore a desirable device that remains to be demonstrated.

In this paper, we demonstrate the first MMI-SA in reflection mode. The all-fiber device consists of a MMF sandwiched in between a SMF and a mirror. The MMI-SA is characterized in the wavelength band of $2000 \mathrm{~nm}$. It has a modulation depth of $12 \%$ and saturation intensity of $18.5 \mathrm{MW} / \mathrm{cm}^{2}$. The operation of the MMI-SA is validated by triggering pulses in a thulium doped fiber laser (TDFL) in linear configuration, leading to mode locking with temporal NLP features of $365 \mathrm{fs}$ and a broad optical bandwidth of $11.2 \mathrm{~nm}$.

\section{SATURABLE ABSORBER}

Figure 1 shows a schematic of the proposed MMI-SA. It consists of a silica-based graded index MMF from Corning, with a core diameter of $62.5 \mu \mathrm{m}$ and arbitrary length of $13 \mathrm{~cm}$. A SMF is spliced to one end of the MMF using UV-epoxy, and simultaneously serves as the input and output of the SA. A silver coated mirror is placed at the other end of the MMF to reflect light back to the MMF and SMF. Silica-based GIMF and SMF are used for the current experiment, but chalcogenide or fluoride-based fibers could be used for the fabrication of an

Arslan Anjum and Martin Rochette are with the Department of Electrical and Computer Engineering at McGill University, Montréal, QC H3A 0E9, Canada (e-mail: arslan.anjum@mail.mcgill.ca; martin.rochette@mcgill.ca). 
MMI-SA compatible with mid-infrared fiber laser operation [17].

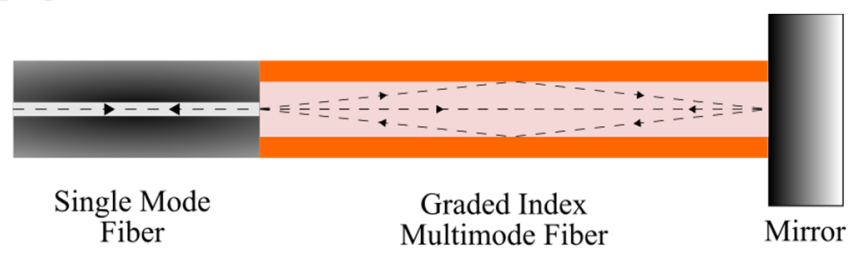

Fig. 1. Nonlinear multimode interference saturable absorber.

Light from the SMF is coupled into various modes of the MMF depending on the lateral alignment between SMF and MMF. These modes travel through the MMF and back from the mirror while accumulating different phase. The power coupled back from the MMF to the SMF is a function of the modal interference pattern at the MMF facet. This interference pattern is a function the input signal power that makes a perturbation on the phase acquired by the modes in the MMF due to selfphase modulation and cross-phase modulation [14]. The lateral offset between SMF and MMF is fixed such that it provides constructive interference for high power input signal, leading to a power dependent reflection coefficient. Hence the structure acts as an artificial saturable absorber.

Figure 2 shows the reflection coefficient of MMI-SA structure measured using a broadband source and optical spectrum analyzer. The broadband reflection spectrum enables the MMI-SA to operate over a broad range of wavelengths compatible with silica glass. The spectrum shows high transmission at wavelengths where the modes of the MMF interfere most constructively at the SMF input. The transmission spectrum can be adjusted with a local maximum to match a target wavelength, by adjustment of the lateral offset between SMF and MMF or by a controlled bending of the MMF [18]. The average coupling loss of the MMI-SA is $\sim 70 \%$, attributed to offset coupling of SMF and MMF.

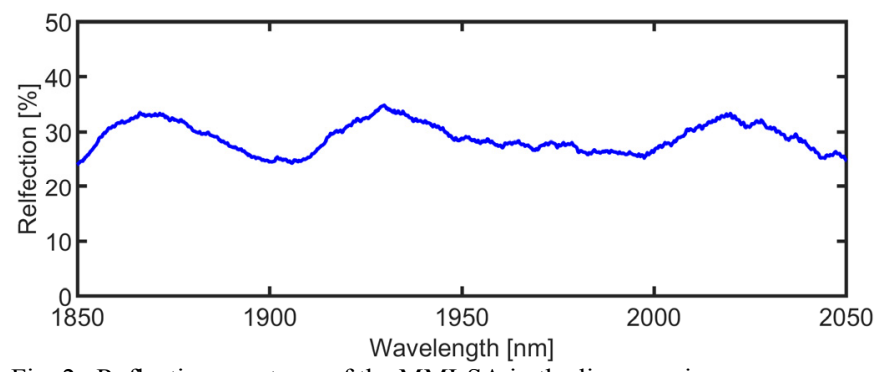

Fig. 2. Reflection spectrum of the MMI-SA in the linear regime.

Figure 3 shows the setup used to measure the nonlinear reflection coefficient of the MMI-SA. Pulses from a mode locked laser (MLL) with a central wavelength of $1937 \mathrm{~nm}$ and duration of $800 \mathrm{fs}$ are sent to the MMI-SA via an optical circulator. A variable optical attenuator is used vary the input power to the MMI-SA. The incident peak power reaching the SA can be varied from 0 to $112 \mathrm{~W}$. After the VOA, pulses from the MLL travel $3 \mathrm{~m}$ in SMF before reaching the SA and acquire a nonlinear phase-shift up to $0.34 \mathrm{rad}$, an amount too low to distort the pulse spectrum significantly. The incident and reflected power of the SA are recorded using calibrated power meters (PM).

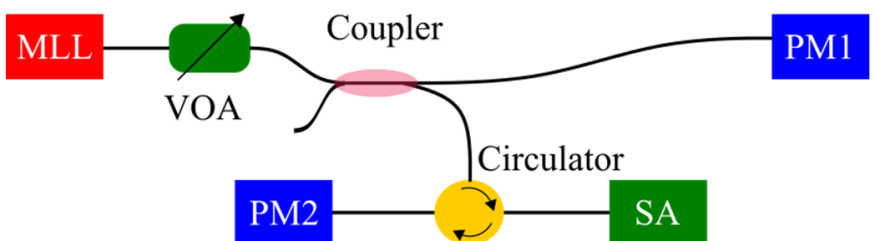

Fig. 3. Characterization setup of the MMI-SA. MLL: Mode-locked laser, VOA: Variable optical attenuator, PM: Power meter.

Figure 4 shows the saturable reflection coefficient profile of the MMI-SA. It is expected that the reflection coefficient profile follows a function given by

$R=1-M \exp \left(-I / I_{\text {sat }}\right)-A_{n s}$, where $M$ is the modulation depth, $A_{n s}$ is the non-saturable loss, and $I_{s a t}$ is the saturation intensity. Experimental data fits perfectly this function with the use of $M=12 \%, A_{n s}=57 \%$ and $I_{\text {sat }}=18.5 \mathrm{MW} / \mathrm{cm}^{2}$. In the current setup, the peak power in the SMF that is required to reach an optical intensity $I_{\text {sat }}$ is $32.7 \mathrm{~W}$.

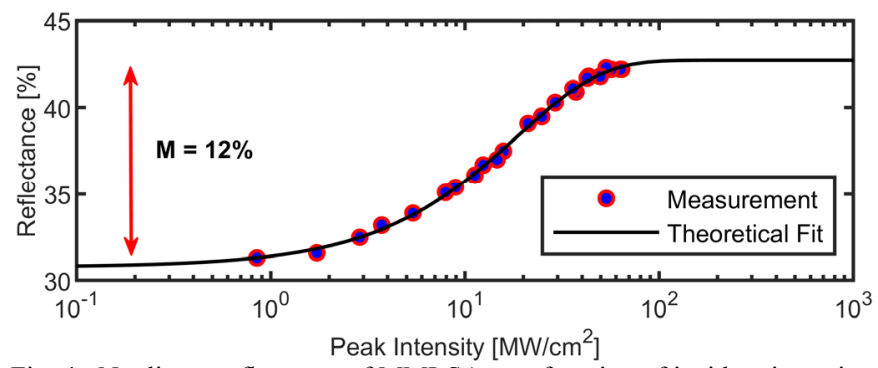

Fig. 4. Nonlinear reflectance of MMI-SA as a function of incident intensity. M: Modulation depth.

\section{LASER CAVITY}

Figure 5 shows a schematic of the TDFL cavity used to validate the functionality of the MMI-SA. The gain is provided by a $23 \mathrm{~cm}$ long thulium-doped fiber (TDF, from Coractive) with a core diameter of $6 \mu \mathrm{m}$ and numerical aperture of 0.24 . The cavity is bounded by a fiber loop mirror (FLM) on one end and the MMI-SA on the other end. The FLM is made from joining the output ports of a 20:80 coupler and provides a reflection coefficient of $50 \%$ at the laser wavelength and it serves as the output port to the fiber laser. A pump laser at a wavelength of $1550 \mathrm{~nm}$ is coupled to the TDF using a $1550 / 1900$ wavelength division multiplexer (WDM). A second WDM is placed after the TDF to remove any residual pump light. An inline polarization controller is used to stabilize the laser oscillation.

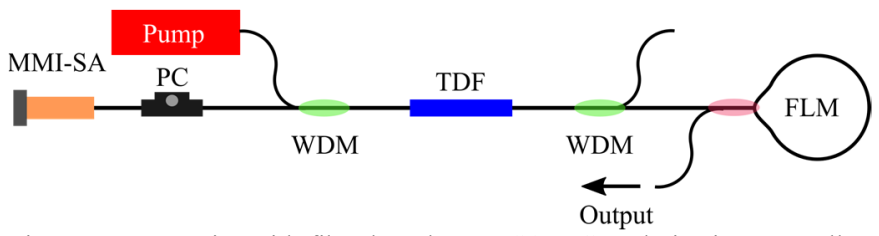

Fig. 5. Laser cavity with fiber based MMI-SA. PC: Polarization controller, WDM: Wavelength division multiplexer, TDF: Thulium doped fiber, FLM: Fiber loop mirror.

The roundtrip cavity length (L) is $29.7 \mathrm{~m}$ and the total cavity dispersion is anomalous with $\beta_{2} \mathrm{~L}=-3.97 \mathrm{ps}^{2}$ at the laser wavelength. When the pump is switched on, the intensity dependent reflection of the MMI-SA results in the formation of 
pulses at a wavelength of $1905 \mathrm{~nm}$. The laser output is observed using a high-speed photodetector that is connected to a realtime oscilloscope with a bandwidth of $350 \mathrm{MHz}$. An electrical spectrum analyzer with a bandwidth of $2 \mathrm{GHz}$ is used to record the RF spectrum from the photodetector. The laser spectrum is monitored using an optical spectrum analyzer with a resolution of $0.1 \mathrm{~nm}$.

\section{RESULTS AND DISCUSSION}

When the pump laser on is switched on, a modelocked oscillation is triggered in the TDFL cavity from the proper adjustment of the polarization controller. Once the position of the polarization controller is fixed at the optimal position, the pulsed laser is also self-starting. Figure 6 shows the TDFL output recorded using the real-time oscilloscope. The pulse to pulse delay is $285 \mathrm{~ns}$, which matches the cavity length of $29.7 \mathrm{~m}$ for single pulse per round trip.

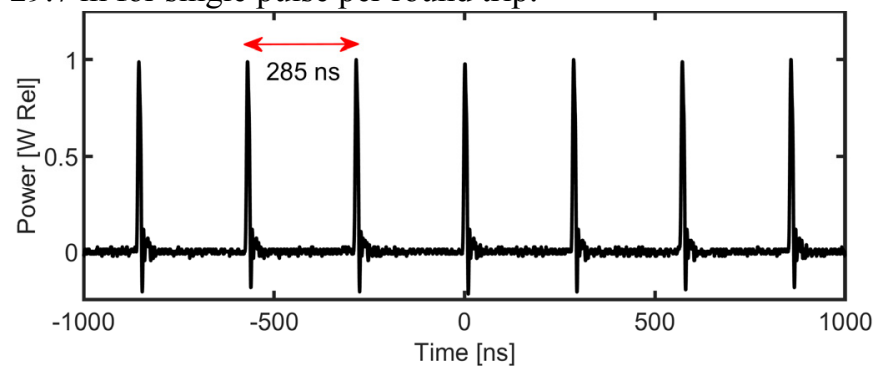

Fig. 6. Pulse train at the fundamental repetition rate.

Figure 7 shows the optical spectrum of the TDFL. The $3 \mathrm{~dB}$ bandwidth of the spectrum is $11.2 \mathrm{~nm}$, which indicates a coherence time of $\sim 345 \mathrm{fs}$. The absence of Kelly sidebands and relatively broad spectrum compared to soliton pulses at similar wavelength [16] is an indication of NLP formation, which is subsequently confirmed by an autocorrelation trace.

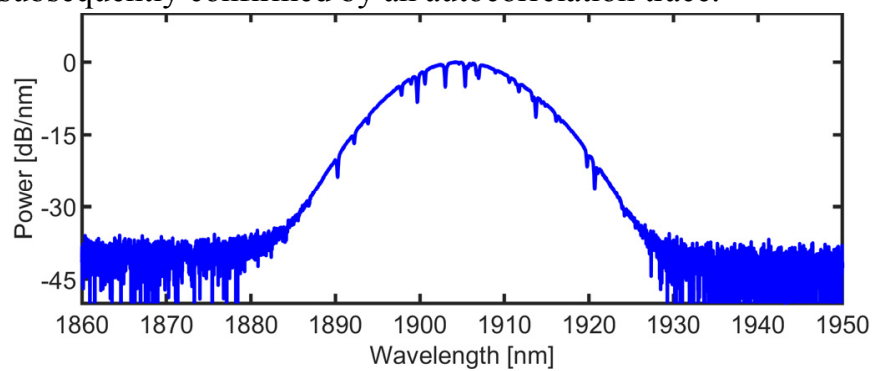

Fig. 7. Optical spectrum of noise like pulses.

Figure 8 (a) shows the autocorrelation trace of the pulses. It shows a sharp peak at zero delay on top of a broad pedestal limited by the temporal range of the autocorrelator. Such an autocorrelation trace is the signature of NLP [9], [19]. Figure 8 (b) is a zoomed in version of the autocorrelation trace close to zero delay. The deconvolved FWHM of the coherent peak is $\sim 365 \mathrm{fs}$, which represents the average duration of pulse features present in the NLP packet. Due to the high GVD $\left(\beta_{2} \mathrm{~L}=-3.97 \mathrm{ps}^{2}\right)$ in the cavity, the cavity soliton breaks into a bunch of narrow pulses with random duration and peak intensity. These pulses are distributed randomly within the NLP packet [20].

Figure 9 (a) shows the RF spectrum of the TDFL output
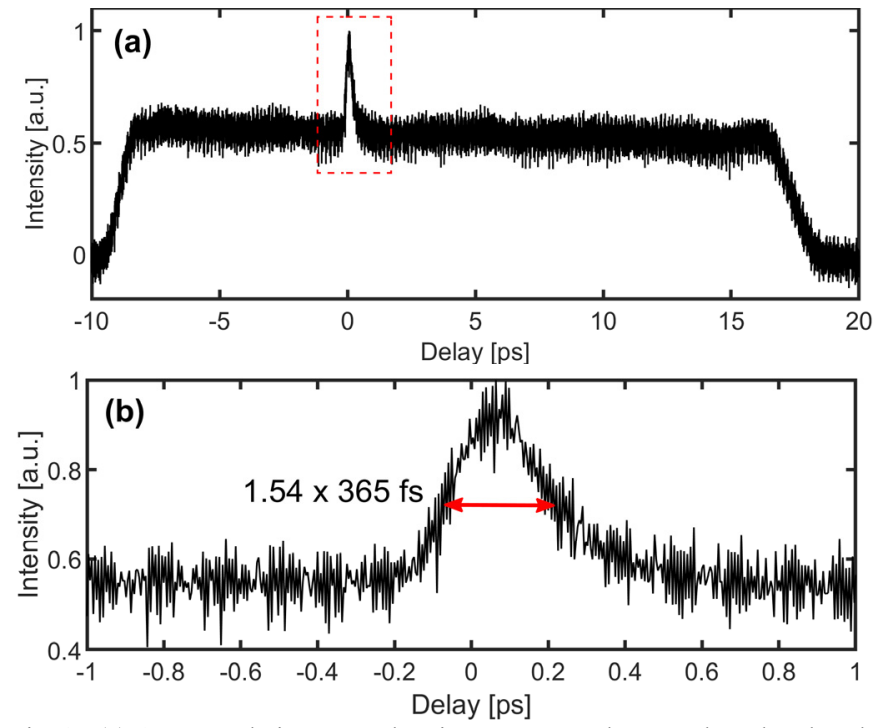

Fig. 8. (a) Autocorrelation trace showing narrow peak over a broad pedestal. (b) The coherent peak close to zero delay.

recorded with a resolution bandwidth of $1 \mathrm{kHz}$. The fundamental peak appears at $3.51 \mathrm{MHz}$, which matches with the pulse to pulse delay of $285 \mathrm{~ns}$. A signal to noise ratio of $57 \mathrm{~dB}$ is observed from the measurement. The high signal to noise ratio and the absence of Q-switching sidebands indicate a stable pulse train. Figure 9 (b) shows the RF spectrum over an extended range of $200 \mathrm{MHz}$. Peaks at the multiple of fundamental repetition rate with a decreasing envelop are observed, which are a signature of stable continuous wave (CW)-modelocked laser.

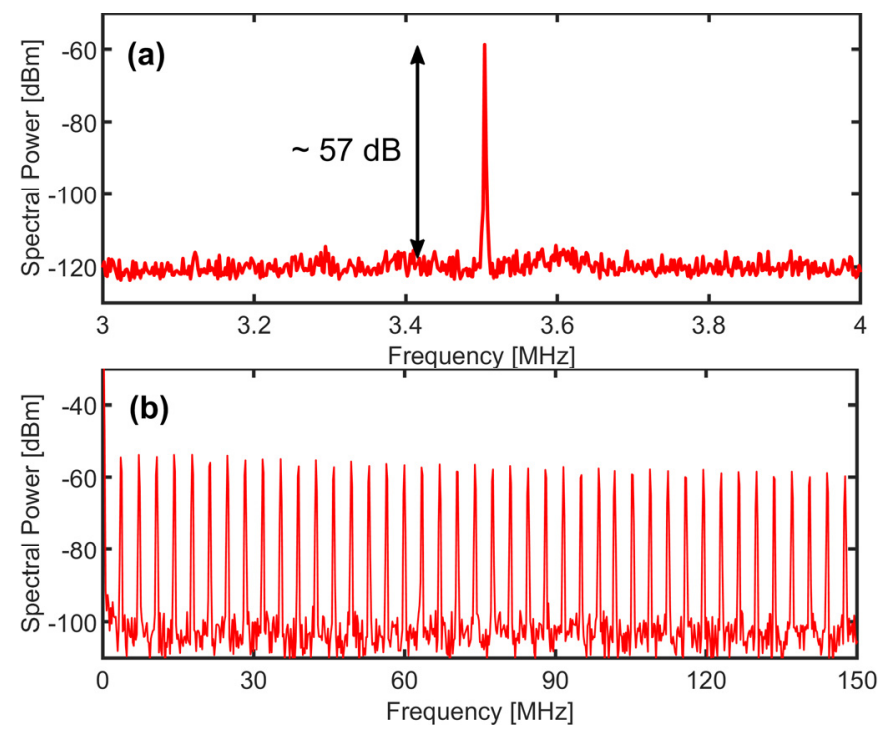

Fig. 9. (a) Fundamental peak of RF spectrum of the TDFL. (b) RF spectrum over $200 \mathrm{MHz}$ frequency span.

This TDFL produces different outputs depending on the pump power as predicted by Ref. [21]. Figure 10 shows the average output power of the laser as a function of the pump power. The TDFL has a threshold of $0.54 \mathrm{~W}$ and a slope efficiency of $1.02 \%$. The maximum output power is $21.9 \mathrm{~mW}$ at pump power of $2.35 \mathrm{~W}$. For a pump power of $0.54-0.70 \mathrm{~W}$, the TDFL produces CW output without pulsation. Between $0.70 \mathrm{~W}$ and $1.3 \mathrm{~W}$, the TDFL operates in Q-switch (QS)- 
modelocking where a pulse train with a varying envelop is produced. Figure 11 (a) shows the QS-ML output recorded at $1 \mathrm{~W}$ of pump power. A QS repetition rate of the envelope is observed at a frequency of $86.8 \mathrm{kHz}$. Beyond $1.3 \mathrm{~W}$ of pump power, the TDFL operates in CW-ML state, where pulses with $\mathrm{CW}$ envelope are generated. In the CW-ML state, the pulse energy is higher than the saturation threshold of the gain medium and the laser is stable against QS-instabilities. Figure 11 (b) shows CW-ML pulses recorded at a pump power of $2 \mathrm{~W}$. The stable pulse train over a broad window of $60 \mu \mathrm{s}$ is consistent with RF-spectrum presented in Figure 9.

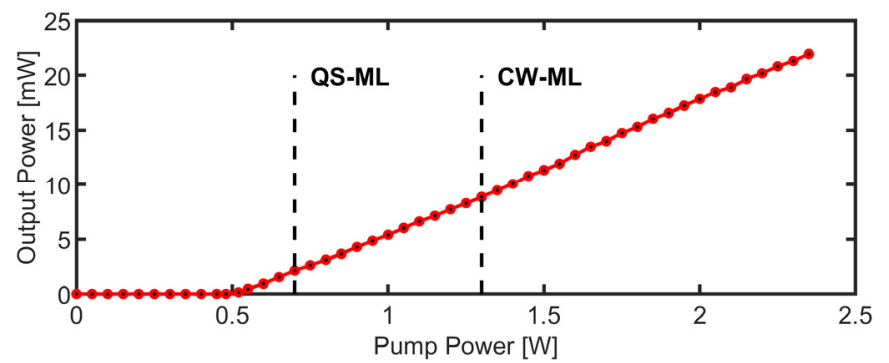

Fig. 10. Average output power of the laser a function of pump power.
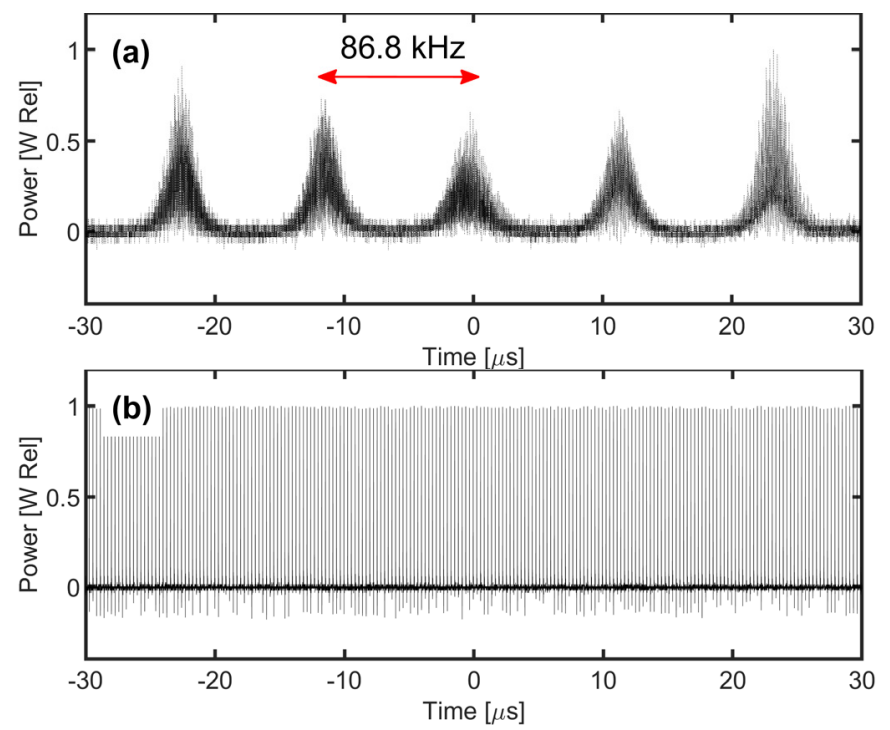

Fig. 11. Laser output in (a) QS modelocked and (b) CW modelocked state respectively.

\section{CONCLUSION}

We have experimentally demonstrated the operation of a reflection mode MMI-SA based on silica graded index multimode fiber. The SA provides a modulation depth of $12 \%$ and generates $\mathrm{CW}$ modelocked pulses in a Tm-doped linear laser cavity without the need of an optical isolator. The laser produces modelocked noise like pulses with a $3 \mathrm{~dB}$ bandwidth of $11.2 \mathrm{~nm}$ at $1905 \mathrm{~nm}$ wavelength. This simple cavity design will pave the way for creation of ultrafast pulsed lasers at longer wavelengths.

\section{REFERENCES}

[1] Q. Wang, J. Geng, T. Luo, and S. Jiang, "Mode-locked $2 \mu \mathrm{m}$ laser with highly thulium-doped silicate fiber" Opt. Lett., vol. 34, no. 23, p. 3616,
Dec. 2009

[2] G. Sobon, J. Sotor, I. Pasternak, A. Krajewska, W. Strupinski, and K. M. Abramski, "Thulium-doped all-fiber laser mode-locked by CVDgraphene/PMMA saturable absorber," Opt. Express, vol. 21, no. 10, p. 12797, 2013.

[3] Q. Wang, T. Chen, B. Zhang, A. P. Heberle, and K. P. Chen, "All-fiber passively mode-locked thulium-doped fiber ring oscillator operated at solitary and noiselike modes," Opt. Lett., vol. 36, no. 19, p. 3750, 2011.

[4] E. Nazemosadat and A. Mafi, "Nonlinear multimodal interference and saturable absorption using a short graded-index multimode optical fiber," J. Opt. Soc. Am. B, vol. 30, no. 5, p. 1357, May 2013.

[5] V. Voropaev et al., "Generation of multi-solitons and noise-like pulses in a high-powered thulium-doped all-fiber ring oscillator," Sci. Rep., vol. 9, no. 1, pp. 1-11, 2019.

[6] S. Chouli and P. Grelu, "Rains of solitons in a fiber laser," Opt. Express, vol. 17, no. 14, p. 11776, 2009.

[7] P. Wang, D. Hu, K. Zhao, L. Jiao, X. Xiao, and C. Yang, "Dissipative Rogue Waves among Noise-Like Pulses in a Tm Fiber Laser Mode Locked by a Monolayer MoS2 Saturable Absorber," IEEE J. Sel. Top. Quantum Electron., vol. 24, no. 3, 2018.

[8] X. Wang et al., "Generation of noise-like pulses with $203 \mathrm{~nm} \mathrm{3-dB}$ bandwidth," Opt. Express, vol. 27, no. 17, p. 24147, 2019.

[9] T. North and M. Rochette, "Raman-induced noiselike pulses in a highly nonlinear and dispersive all-fiber ring laser," Opt. Lett., vol. 38, no. 6, p. 890, 2013.

[10] S. Keren and M. Horowitz, "Interrogation of fiber gratings by use of lowcoherence spectral interferometry of noiselike pulses," Opt. Lett., vol. 26, no. 6, p. 328, 2001.

[11] S. Keren, E. Brand, Y. Levi, B. Levit, and M. Horowitz, "Data storage in optical fibers and reconstruction by use of low-coherence spectral interferometry," Opt. Lett., vol. 27, no. 2, p. 125, Jan. 2002.

[12] A. Zaytsev, C.-H. Lin, Y.-J. You, C.-C. Chung, C.-L. Wang, and C.-L. Pan, "Supercontinuum generation by noise-like pulses transmitted through normally dispersive standard single-mode fibers," Opt. Express, vol. 21, no. 13, p. 16056, Jul. 2013.

[13] K. Özgören, B. Öktem, S. Yilmaz, F. Ö. Ilday, and K. Eken, " 83 W, 31 $\mathrm{MHz}$, square-shaped, 1 ns-pulsed all-fiber-integrated laser for micromachining," Opt. Express, vol. 19, no. 18, p. 17647, Aug. 2011.

[14] T. Chen, Q. Zhang, Y. Zhang, X. Li, H. Zhang, and W. Xia, "All-fiber passively mode-locked laser using nonlinear multimode interference of step-index multimode fiber," Photonics Res., vol. 6, no. 11, p. 1033, Nov. 2018.

[15] Z. Wang et al., "Er-Doped Mode-Locked Fiber Laser with a Hybrid Structure of a Step-Index-Graded-Index Multimode Fiber as the Saturable Absorber," J. Light. Technol., vol. 35, no. 24, pp. 5280-5285, 2017.

[16] H. Li, Z. Wang, C. Li, J. Zhang, and S. Xu, "Mode-locked Tm fiber laser using SMF-SIMF-GIMF-SMF fiber structure as a saturable absorber," Opt. Express, vol. 25, no. 22, p. 26546, 2017.

[17] K. Zhang and M. Rochette, "All-Fiber Saturable Absorber Using Nonlinear Multimode Interference in a Chalcogenide Fiber," J. Light. Technol., vol. 38, no. 22, pp. 6321-6326, Nov. 2020.

[18] K. Zhang, I. Alamgir, and M. Rochette, "Midinfrared Compatible Tunable Bandpass Filter Based on Multimode Interference in Chalcogenide Fiber," J. Light. Technol., vol. 38, no. 4, pp. 857-863, Feb. 2020.

[19] M. Horowitz, Y. Barad, and Y. Silberberg, "Noiselike pulses with a broadband spectrum generated from an erbium-doped fiber laser," Opt. Lett., vol. 22, no. 11, p. 799, 1997.

[20] J. C. Hernandez-Garcia, O. Pottiez, J. M. Estudillo-Ayala, and R. RojasLaguna, "Numerical analysis of a broadband spectrum generated in a standard fiber by noise-like pulses from a passively mode-locked fiber laser," Opt. Commun., vol. 285, no. 7, pp. 1915-1919, 2012.

[21] C. Hönninger, R. Paschotta, F. Morier-Genoud, M. Moser, and U. Keller, "Q-switching stability limits of continuous-wave passive mode locking," J. Opt. Soc. Am. B, vol. 16, no. 1, p. 46, Jan. 1999. 